\title{
HRM IN PRIVATE AND PUBLIC ORGANIZATIONS IN SERBIA
}

\author{
Nemanja BERBER ${ }^{1}$, Agneš SLAVIĆ ${ }^{2}$ \\ ${ }^{1}$ University of Novi Sad, Faculty of Economics, 24000 Subotica, Segedinski put 9-11, Republic of Serbia \\ E-mail: berber@ef.uns.ac.rs \\ ${ }^{2}$ University of Novi Sad, Faculty of Economics, 24000 Subotica, Segedinski put 9-11, Republic of Serbia
}

Paper received: 29.04.2016.; Paper accepted: 25.05.2016.

\begin{abstract}
Human resource management (HRM) is a management concept which obtains many practices and activities. Although there is a substantial literature on HRM in the private sector, the practice of HRM in the public sector is still scarce. In the private sector, HRM is found as a factor for gaining competitive advantage, especially if HR practices are implemented in the way of highperforming working practice (HPWP). In the public sector, HRM is seen as paternalistic management, with the standardization of employment practices, collective bargaining and working practices that emphasize equal opportunities for employees. The goal of this research is to explore the characteristics and differences between HRM practice in organizations from the private and public sector. The subject of the research is HRM practice (staffing, training and development, compensation and benefits, and industrial relation and communication) in the private and public organizations in the Republic of Serbia. The methodology of the paper includes exploration of the available literature on the theme and statistical analysis of the differences between HR practices in organizations from the private and public sector. The research is based on the HR data gathered in the second CRANET research round in Serbia, performed in 2015.
\end{abstract}

Keywords: Human resource management, Private sector, public sector, Serbia, Cranet.

\section{INTRODUCTION}

HRM as a concept of managing human capital is widely explored and discussed in private sector organizations. Most of the themes are related to the exploration of HRM activities (HR planning, staffing, training and development, compensation, retention, communication) in large private organizations, where HRM is found as a factor for gaining competitive advantage, especially if HR practices are implemented in the way of 'high performance', 'high commitment', or 'high involvement' practices as they are "thought to release untapped reserves of 'human resourcefulness' by increasing employee commitment, participation and involvement" (Gould-Williams, 2004). On the other side, there is a lack of the literature and empirical research in the area of HRM in small and medium enterprises (Urbano and Yordanova, 2008; Zolak-Poljašević and Petković, 2013; Štangl Šušnjar et al., 2016) and organizations from public sector (Boyne et al.,
1999; Gould-Williams, 2004; Marčetić, 2006; Giauque et al., 2013). In the public sector, in the UK for example, HRM is usually seen as paternalistic management, with the standardization of employment practices, collective bargaining and working practices that emphasize equal opportunities for employees (Boyne et al., 1999; Gould-Williams, 2004).

For the purpose of this paper, we decided to explore the practice of HRM in the public sector organizations in comparison with the HRM in the private sector organizations. The goal of this research is to explore the characteristics and differences between HRM practice in organizations from the private and public sector. The subject of the research is HRM practice (staffing, training and development, compensation and benefits, industrial relation and communication) in the private and public sector organizations in the Republic of Serbia. The methodology of the paper includes exploration of 
the available literature on the theme and statistical analysis of the differences between HR practices in organizations from the private and public sector. The research is based on the HR data gathered in the second CRANET research ${ }^{1}$ round, performed in Serbia in 2015.

\section{THEORETICAL BACKGROUND}

The main goal of the enterprises from the private sector is a long-term business success and earnings for shareholders. In the case of public sector enterprises, there are two purposes of business: the provision of general public interest and achieving the commercial objectives of the business, i.e. economic benefits (Mijić et al., 2015). Public sector is seen as less successful in comparison with private sector organizations regarding performances and management approaches (Mijić et al., 2015; Caemmerer and Dewar, 2013; Boubakri and Cosset, 1998; Megginson et al., 1994). Today, each and every organization has to manage all its resources in the best way to create new value if it wants to survive. Therefore it was interesting to explore HRM approach in public and private sector since HRM is one of the driving forces for competitive advantages in modern business (Berber and Slavić, 2014; Campbell et al., 2012; Wright and McMahan, 2011).

In the traditional model of the public sector organizations, employment policy was based on bureaucratic practices and principles of rulegoverned rational action. "The administrative system was subjected to a bureaucratization of procedures to ensure that decisions and actions were consistent, formalized and systematically addressed activities through a pre-defined application of rules and processes" (Brown, 2008). The employment system in public sector can be described as (Štangl Šušnjar, 2013; Chaston, 2011; Brown, 2008; Marčetić, 2006; Boyne et al., 1999):

- Highly centralized and run by powerful state agencies or ministries that are responsible for all HRM decisions - staffing, training and career development, compensation, retirement.

- Compensation is based on the job position and/or seniority.

- Job positions are narrow, specific task-based and highly routinized, usually outdated.

\footnotetext{
${ }^{1}$ http://www.ef.uns.ac.rs/cranet/index.html
}

- Seniority or length in the service was the basis for promotion and career development.

- The influence of trade unions is usually strong.

- Since the main goal of public sector organizations is the provision of public service, in addition to economic, public sector organizations must achieve other legal, democratic and social values.

- Public organizations had significant financial help from local, regional or state level.

- Management and organization of public sector organization are under the pressure of wide range of political and economic factors.

This kind of organizational systems came under strong pressure of the modern business conditions, especially economic recession. The new approach to management in public sector organizations should allow greater flexibility in dealing with HRM issues, which would make the transformation from traditional, transactional HRM to strategic HRM, oriented to the increase of the employees' productivity and organizational outcomes. One possible approach is the New Public Management, described as a more flexible approach to public management in terms of performance-based organization which facilitates innovation and efficiency of public enterprises, and personal responsibility of managers (Štangl Šušnjar, 2013).

Main determinants of the reform of public sector management lie in (Hughes, 2003):

- strategic approach;

- management, not administration;

- focus on results;

- improvement of financial management;

- flexibility in staffing;

- flexibility in the organization;

- the shift to greater competition;

- new contractualism;

- stressing private management styles and practices;

- separation of purchasers and providers;

- re-examination of the role of the government.

Since the transformation of public management is a very complex issue, we decided to explore only one segment of management in private and public sector organizations, HRM practice. Our research hypothesis, based on the above presented theoretical sources and past researches, is: 
H1: Human resource management practice is less developed in public sector than in private sector organizations in Serbia.

\section{METHODOLOGY}

The applied methodology of the survey was formulated and has been developed by the research fellows of CRANET (Cranfield Network on Comparative Human Resource Management) network founded by five countries in 1989. It has become the basis of regular comparative surveys on HR policy and practice in Europe and in an increasing number of countries worldwide. The survey is conducted approximately every four years (Steinmetz et al., 2011) in over 40 countries of the world (Lazarova et al., 2008). The aim of the research is to provide high-quality data for the purposes of academic, public and private sectors, as well as for students of human resource management, and to create new knowledge about human resource management practices in different countries of the world. The questionnaire was divided into six parts/sections: HRM activities in organization, staffing, employee development, compensation and benefits, industrial relations and communication, and organizational details. Despite some methodological limitations, Cranet studies have brought important empirical data since 1990, providing insights into the development of human resource management practices in member countries, whose number is growing steadily, and to the theoretical development of the field of comparative human resource management (Karoliny et al., 2009).

Faculty of Economics in Subotica has performed two cycles of research on the practice of human resource management in Serbia according to the methodology of international Cranet research. As the only member of the international scientific network from Serbia, Faculty of Economics participated in this international examination of the activities of HRM in 2008 for the first time with the 50 analyzed organizations. In the first half of 2015, we examined 160 organizations. This was the second cycle of the Cranet research in Serbia. The answers to the questions gave HR managers in organizations (Leković et al., 2015).

Regarding the sample, the largest share of the sample in Serbia in 2015 was SME sector, $60 \%$. There are $27 \%$ of large organizations and $13 \%$ of very large, with more than 1000 employees. The sample of explored organizations in Serbia consisted of public (34\%) and private (66\%) sector. Around $8 \%$ of analyzed organizations are from the agriculture sector, $35 \%$ is from industry sector, and $57 \%$ of organizations are from the service sector. We examined the main differences in the basic HRM activities (recruitment, selection, training, career development, compensation, industrial relation, and communication) between organizations from private and public sector. We used non-parametric statistical techniques (Spearman's Chi Square test and Mann-Whitney U test). Main techniques of recruitment, selection, training, development, compensation and communication were taken from Cranet database, in the form of variables (dummies and continuous). We used Chi Square test to explore the differences between categorical variables and Mann-Whitney test in case of continuous variables.

\section{RESULTS}

For the analysis of the differences between organizations from private and public sector in the usage of contemporary recruitment and selection techniques, the authors used Spearman Chi Square test. According to the data from table 1, there are statistically significant differences between private and public organizations in the usage of recruitment and selection methods.

Generally, organizations from public sector use modern techniques of recruitment and selection for professional workers less than those from the private sector $(p<0.05)$. This is obvious for all techniques of recruitment, except for internal recruitment, where we found no statistically significant differences $(\mathrm{p}=0.99)$. The strengths of the associations between two variables were weak (Phi from 0.217 to 0.429). Similar results are found for the selection techniques. In the case of psychometric, online, and ability tests no statistically significant $(p>0.05)$ differences were found, while other techniques are used in a higher share of organizations from private than from public sector.

We used Mann-Whitney $U$ test to explore the differences between organizations from private and public sector regarding the usage of training techniques. According to the data from table 2 and 3 , there are statistically significant differences between private and public sector regarding the number of days spent on training for managers and manual workers. Private sector organizations use more days than public sector organizations for 
N. Berber and A. Slavić
HRM in private and public organizations in Serbia these two categories of employees. In the case of professional workers and the percentage of payroll costs used for training, there were no statistically significant differences found.

Table 1: Differences between private and public organizations in recruitment and selection practice

\begin{tabular}{|c|c|c|c|c|c|c|c|c|c|}
\hline \multicolumn{5}{|c|}{\begin{tabular}{|c|} 
Recruitment \\
\end{tabular}} & \multicolumn{5}{|c|}{ Selection } \\
\hline & \multicolumn{2}{|c|}{ Internal recruitment } & \multirow{2}{*}{$\begin{array}{c}\text { Pearson Chi- } \\
\text { Square } \\
\text { Asymp. Sig. } \\
\text { (2-sided) }\end{array}$} & \multirow{2}{*}{$\begin{array}{c}2,714 \\
, 099\end{array}$} & & \multicolumn{2}{|c|}{ Interview panel } & \multirow{2}{*}{\begin{tabular}{|c|}
$\begin{array}{c}\text { Pearson Chi- } \\
\text { Square }\end{array}$ \\
$\begin{array}{c}\text { Asymp. Sig. } \\
\text { (2-sided) }\end{array}$ \\
\end{tabular}} & \multirow{2}{*}{$\begin{array}{r}4,248 \\
, 039\end{array}$} \\
\hline & $\begin{array}{l}\text { Generally } \\
\text { not used }\end{array}$ & Yes & & & & $\begin{array}{c}\text { Generally } \\
\text { not used }\end{array}$ & Yes & & \\
\hline $\begin{array}{l}\text { Private } \\
\text { sector }\end{array}$ & $39,0 \%$ & $61,0 \%$ & Phi &, 131 & $\begin{array}{l}\text { Private } \\
\text { sector }\end{array}$ & $45,7 \%$ & $54,3 \%$ & Phi &, 163 \\
\hline $\begin{array}{l}\text { Public } \\
\text { sector }\end{array}$ & $25,9 \%$ & $74,1 \%$ & & & $\begin{array}{l}\text { Public } \\
\text { sector }\end{array}$ & $63,0 \%$ & $37,0 \%$ & & \\
\hline \multirow[t]{3}{*}{ Total } & $34,6 \%$ & $65,4 \%$ & & & Total & $51,6 \%$ & $48,4 \%$ & & \\
\hline & \multicolumn{2}{|c|}{$\begin{array}{c}\text { Word of mouth/employee } \\
\text { referral }\end{array}$} & $\begin{array}{l}\text { Pearson Chi- } \\
\text { Square }\end{array}$ & 7,487 & & \multicolumn{2}{|c|}{ Psychometric test } & $\begin{array}{l}\text { Pearson Chi- } \\
\text { Square }\end{array}$ & 0,125 \\
\hline & $\begin{array}{c}\text { Generally } \\
\text { not used }\end{array}$ & Yes & $\begin{array}{l}\text { Asymp. Sig. } \\
\text { (2-sided) }\end{array}$ & ,006 & & $\begin{array}{c}\text { Generally } \\
\text { not used }\end{array}$ & Yes & $\begin{array}{l}\text { Asymp. Sig. } \\
\text { (2-sided) }\end{array}$ & ,724 \\
\hline $\begin{array}{l}\text { Private } \\
\text { sector }\end{array}$ & $41,9 \%$ & $58,1 \%$ & Phi &, 217 & $\begin{array}{l}\text { Private } \\
\text { sector }\end{array}$ & $71,4 \%$ & $28,6 \%$ & Phi &, 028 \\
\hline $\begin{array}{l}\text { Public } \\
\text { sector }\end{array}$ & $64,8 \%$ & $35,2 \%$ & & & $\begin{array}{l}\text { Public } \\
\text { sector }\end{array}$ & $74,1 \%$ & $25,9 \%$ & & \\
\hline \multirow[t]{3}{*}{\begin{tabular}{|l|} 
Total \\
\end{tabular}} & $49,7 \%$ & $50,3 \%$ & & & Total & $72,3 \%$ & $27,7 \%$ & & \\
\hline & \multicolumn{2}{|c|}{ Social media } & $\begin{array}{c}\text { Pearson Chi- } \\
\text { Square }\end{array}$ & 11,189 & & \multicolumn{2}{|c|}{ Assessment centre } & $\begin{array}{c}\text { Pearson Chi- } \\
\text { Square }\end{array}$ & 7,15 \\
\hline & $\begin{array}{l}\text { Generally } \\
\text { not used }\end{array}$ & Yes & $\begin{array}{l}\text { Asymp. Sig. } \\
\text { (2-sided) }\end{array}$ &, 001 & & \begin{tabular}{|c|} 
Generally \\
not used
\end{tabular} & Yes & $\begin{array}{l}\text { Asymp. Sig. } \\
\text { (2-sided) }\end{array}$ & ,007 \\
\hline $\begin{array}{l}\text { Private } \\
\text { sector }\end{array}$ & $78,1 \%$ & $21,9 \%$ & Phi & ,265 & $\begin{array}{l}\text { Private } \\
\text { sector }\end{array}$ & $72,4 \%$ & $27,6 \%$ & Phi & ,212 \\
\hline $\begin{array}{l}\text { Public } \\
\text { sector }\end{array}$ & $98,1 \%$ & $1,9 \%$ & & & $\begin{array}{l}\text { Public } \\
\text { sector }\end{array}$ & $90,7 \%$ & $9,3 \%$ & & \\
\hline \multirow[t]{3}{*}{ Total } & $84,9 \%$ & $15,1 \%$ & & & \begin{tabular}{|l|} 
Total \\
\end{tabular} & $78,6 \%$ & $21,4 \%$ & & \\
\hline & \multicolumn{2}{|c|}{ Career fairs } & $\begin{array}{l}\text { Pearson Chi- } \\
\text { Square }\end{array}$ & 14,48 & & \multicolumn{2}{|c|}{ Social media profiles } & $\begin{array}{l}\text { Pearson Chi- } \\
\text { Square }\end{array}$ & 9,149 \\
\hline & $\begin{array}{l}\text { Generally } \\
\text { not used }\end{array}$ & Yes & $\begin{array}{l}\text { Asymp. Sig. } \\
\text { (2-sided) }\end{array}$ &, 000 & & \begin{tabular}{|c|} 
Generally \\
not used
\end{tabular} & Yes & $\begin{array}{c}\text { Asymp. Sig. } \\
\text { (2-sided) }\end{array}$ & ,002 \\
\hline $\begin{array}{l}\text { Private } \\
\text { sector }\end{array}$ & $57,1 \%$ & $42,9 \%$ & Phi &, 302 & $\begin{array}{l}\text { Private } \\
\text { sector }\end{array}$ & $84,8 \%$ & $15,2 \%$ & Phi & ,240 \\
\hline \begin{tabular}{|l|} 
Public \\
sector \\
\end{tabular} & $87,0 \%$ & $13,0 \%$ & & & \begin{tabular}{|l|} 
Public \\
sector \\
\end{tabular} & $100,0 \%$ & & & \\
\hline \multirow[t]{3}{*}{ Total } & $67,3 \%$ & $32,7 \%$ & & & \begin{tabular}{|l|} 
Total \\
\end{tabular} & $89,9 \%$ & $10,1 \%$ & & \\
\hline & \multicolumn{2}{|c|}{$\begin{array}{c}\text { Recruitment } \\
\text { agencies }\end{array}$} & $\begin{array}{l}\text { Pearson Chi- } \\
\text { Square }\end{array}$ & 9,149 & & \multicolumn{2}{|c|}{ References selection } & $\begin{array}{l}\text { Pearson Chi- } \\
\text { Square }\end{array}$ & 7,59 \\
\hline & $\begin{array}{c}\text { Generally } \\
\text { not used }\end{array}$ & Yes & $\begin{array}{l}\text { Asymp. Sig. } \\
\text { (2-sided) }\end{array}$ &, 002 & & $\begin{array}{c}\text { Generally } \\
\text { not used }\end{array}$ & Yes & $\begin{array}{l}\text { Asymp. Sig. } \\
\text { (2-sided) }\end{array}$ & ,006 \\
\hline \begin{tabular}{|l|} 
Private \\
sector
\end{tabular} & $84,8 \%$ & $15,2 \%$ & Phi & ,240 & \begin{tabular}{|l|} 
Private \\
sector
\end{tabular} & $24,8 \%$ & $75,2 \%$ & Phi & ,218 \\
\hline $\begin{array}{l}\text { Public } \\
\text { sector }\end{array}$ & $100,0 \%$ & & & & \begin{tabular}{|l|} 
Public \\
sector
\end{tabular} & $46,3 \%$ & $53,7 \%$ & & \\
\hline \multirow[t]{3}{*}{ Total } & $89,9 \%$ & $10,1 \%$ & & & Total & $32,1 \%$ & $67,9 \%$ & & \\
\hline & \multicolumn{2}{|c|}{$\begin{array}{l}\text { Vacancy page on } \\
\text { company website }\end{array}$} & $\begin{array}{c}\text { Pearson Chi- } \\
\text { Square }\end{array}$ & 14,815 & & \multicolumn{2}{|c|}{$\begin{array}{c}\text { Ability tests/ Work } \\
\text { sample }\end{array}$} & $\begin{array}{c}\text { Pearson Chi- } \\
\text { Square }\end{array}$ & 2,969 \\
\hline & $\begin{array}{c}\text { Generally not } \\
\text { used }\end{array}$ & Yes & $\begin{array}{l}\text { Asymp. Sig. } \\
\text { (2-sided) }\end{array}$ &, 000 & & \begin{tabular}{|c|} 
Generally \\
not used
\end{tabular} & Yes & $\begin{array}{l}\text { Asymp. Sig. } \\
\text { (2-sided) }\end{array}$ & 085 \\
\hline $\begin{array}{l}\text { Private } \\
\text { sector }\end{array}$ & $40,0 \%$ & $60,0 \%$ & Phi &, 305 & $\begin{array}{l}\text { Private } \\
\text { sector }\end{array}$ & $48,6 \%$ & $51,4 \%$ & Phi &, 137 \\
\hline $\begin{array}{l}\text { Public } \\
\text { sector }\end{array}$ & $72,2 \%$ & $27,8 \%$ & & & $\begin{array}{l}\text { Public } \\
\text { sector }\end{array}$ & $63,0 \%$ & $37,0 \%$ & & \\
\hline \multirow[t]{3}{*}{ Total } & $50,9 \%$ & $49,1 \%$ & & & Total & $53,5 \%$ & $46,5 \%$ & & \\
\hline & \multicolumn{2}{|c|}{$\begin{array}{c}\text { Vacancies on } \\
\text { commercial job websites }\end{array}$} & $\begin{array}{l}\text { Pearson Chi- } \\
\text { Square }\end{array}$ & 29,289 & & \multicolumn{2}{|c|}{ Online selection tests } & $\begin{array}{l}\text { Pearson Chi- } \\
\text { Square }\end{array}$ & 0,199 \\
\hline & $\begin{array}{c}\text { Generally } \\
\text { not used }\end{array}$ & Yes & $\begin{array}{l}\text { Asymp. Sig. } \\
\text { (2-sided) }\end{array}$ &, 000 & & $\begin{array}{c}\text { Generally } \\
\text { not used } \\
\end{array}$ & Yes & $\begin{array}{l}\text { Asymp. Sig. } \\
\text { (2-sided) }\end{array}$ & 0,656 \\
\hline \begin{tabular}{|l|} 
Private \\
sector
\end{tabular} & $36,2 \%$ & $63,8 \%$ & Phi & ,429 & $\begin{array}{l}\text { Private } \\
\text { sector }\end{array}$ & $90,5 \%$ & $9,5 \%$ & Phi & ,035 \\
\hline $\begin{array}{l}\text { Public } \\
\text { sector }\end{array}$ & $81,5 \%$ & $18,5 \%$ & & & $\begin{array}{l}\text { Public } \\
\text { sector }\end{array}$ & $92,6 \%$ & $7,4 \%$ & & \\
\hline Total & $51,6 \%$ & $48,4 \%$ & & & Total & $91,2 \%$ & $8,8 \%$ & & \\
\hline
\end{tabular}

Source: Authors' analysis based on Cranet database 
We used Mann-Whitney $U$ test to explore the differences between organizations from private and public sector regarding the usage of career management techniques $(0=$ not used to $4=$ used to a large extent). According to the data from table 4 and 5 , there are statistically significant differences between private and public sector regarding several techniques for career development $(p<0.05)$. Private sector organizations use most of the listed career development techniques in a greater extent than organizations from the public sector (Means). Public sector organizations use modern techniques at very low level.

Table 2: Differences between private and public organizations in training practice

\begin{tabular}{|l|l|c|c|}
\hline & Sector & Mean Rank & Sum of Ranks \\
\hline \multirow{2}{*}{$\begin{array}{l}\text { Percentage of the annual payroll } \\
\text { costs spent on training }\end{array}$} & Private sector & 83.23 & 8656.00 \\
\cline { 2 - 4 } & Public sector & 72.31 & 3905.00 \\
\cline { 2 - 4 } & Total & & \\
\hline \multirow{2}{*}{$\begin{array}{l}\text { Approximate number of days } \\
\text { Managers receive training }\end{array}$} & Private sector & 81.26 & 7800.50 \\
\cline { 2 - 4 } & Public sector & 65.27 & 3524.50 \\
\cline { 2 - 4 } & Total & & 7520.50 \\
\hline \multirow{2}{*}{$\begin{array}{l}\text { Approximate number of days } \\
\text { Professionals receive training }\end{array}$} & Private sector & 77.53 & 3955.50 \\
\cline { 2 - 4 } & Public sector & 73.25 & 7918.50 \\
\cline { 2 - 4 } & Total & & 3557.50 \\
\hline $\begin{array}{l}\text { Approximate number of days } \\
\text { Clericals/Manuals receive } \\
\text { training }\end{array}$ & Private sector & 81.63 & \\
\cline { 2 - 4 } & Public sector & 65.88 & \\
\cline { 2 - 4 } & Total & & \\
\hline
\end{tabular}

Source: Authors' analysis based on Cranet database

Table 3: Mann - Whitney U test and Means for differences between private and public organizations in training practice

\begin{tabular}{|l|c|c|c|c|}
\hline \multicolumn{5}{|c|}{ Test Statistics } \\
\hline & $\begin{array}{c}\text { Percentage of } \\
\text { the annual } \\
\text { payroll costs } \\
\text { spent on training }\end{array}$ & $\begin{array}{c}\text { Approximate } \\
\text { number of days } \\
\text { Managers } \\
\text { receive training }\end{array}$ & $\begin{array}{c}\text { Approximate } \\
\text { number of days } \\
\text { Professionals } \\
\text { receive training }\end{array}$ & $\begin{array}{c}\text { Approximate } \\
\text { number of days } \\
\text { Clericals/Manu } \\
\text { als receive } \\
\text { training }\end{array}$ \\
\hline Private sector mean & 2.85 & 7.23 & 6.65 & 5.40 \\
\hline Public sector mean & 2.31 & 5.54 & 5.94 & 3.65 \\
\hline Total mean & 2.66 & 6.62 & 6.40 & 4.77 \\
\hline Mann-Whitney U & 2420.000 & 2039.500 & 2470.500 & 2072.500 \\
\hline Asymp. Sig. (2-tailed) & .143 & $\mathbf{. 0 2 8}$ & .554 & $\mathbf{. 0 3 2}$ \\
\hline a. Grouping Variable: Sector & \multicolumn{4}{|l}{} \\
\hline
\end{tabular}

Source: Authors' analysis based on Cranet database

Table 4: Differences between private and public organizations in career development practice

\begin{tabular}{|c|c|c|c|c|c|c|c|}
\hline & Sector & $\begin{array}{l}\text { Mean } \\
\text { Rank }\end{array}$ & $\begin{array}{l}\text { Sum of } \\
\text { Ranks }\end{array}$ & & Sector & $\begin{array}{l}\text { Mean } \\
\text { Rank }\end{array}$ & $\begin{array}{l}\text { Sum of } \\
\text { Ranks }\end{array}$ \\
\hline \multirow{2}{*}{$\begin{array}{l}\text { Use of } \\
\text { Special tasks }\end{array}$} & Private sector & 83.63 & 8614 & \multirow{2}{*}{\begin{tabular}{|l|} 
Use of \\
Succession \\
plans
\end{tabular}} & Private sector & 86.18 & 8876.5 \\
\hline & Public sector & 70.17 & 3789 & & \begin{tabular}{|l|} 
Public sector \\
\end{tabular} & 65.31 & 3526.5 \\
\hline \multirow{3}{*}{$\begin{array}{l}\text { Use of Projects } \\
\text { to stimulate } \\
\text { learning }\end{array}$} & Private sector & 81.11 & 8273 & \multirow{3}{*}{$\begin{array}{l}\text { Use of } \\
\text { Planned job } \\
\text { rotation }\end{array}$} & Private sector & 87.27 & 9076.5 \\
\hline & Public sector & 73.57 & 3973 & & \begin{tabular}{|l|} 
Public sector \\
\end{tabular} & 62.76 & 3326.5 \\
\hline & & & & & & & \\
\hline \multirow{3}{*}{$\begin{array}{l}\text { Use of Training } \\
\text { on-the-job }\end{array}$} & Private sector & 85.44 & 8800 & \multirow{3}{*}{$\begin{array}{l}\text { Use of } \\
\text { "High flier" } \\
\text { schemes }\end{array}$} & Private sector & 86.19 & 8877.5 \\
\hline & Public sector & 66.72 & 3603 & & \begin{tabular}{|l|} 
Public sector \\
\end{tabular} & 63.56 & 3368.5 \\
\hline & & & & & & & \\
\hline \multirow{3}{*}{$\begin{array}{l}\text { Use of } \\
\text { Participation in } \\
\text { project team work }\end{array}$} & Private sector & 80.09 & 8249.5 & \multirow{3}{*}{$\begin{array}{l}\text { Use of } \\
\text { International } \\
\text { work assignments }\end{array}$} & Private sector & 86.59 & 8919 \\
\hline & Public sector & 76.92 & 4153.5 & & Public sector & 64.52 & 3484 \\
\hline & & & & & & & \\
\hline \multirow{3}{*}{$\begin{array}{l}\text { Use of Formal } \\
\text { networking } \\
\text { schemes }\end{array}$} & Private sector & 81.18 & 8361.5 & \multirow{3}{*}{$\begin{array}{l}\text { Use of } \\
\text { Coaching }\end{array}$} & Private sector & 87.74 & 9037 \\
\hline & \begin{tabular}{|l|} 
Public sector \\
\end{tabular} & 74.84 & 4041.5 & & \begin{tabular}{|l|} 
Public sector \\
\end{tabular} & 62.33 & 3366 \\
\hline & & & & & Total & & \\
\hline \multirow{2}{*}{$\begin{array}{l}\text { Use of Formal } \\
\text { career plans }\end{array}$} & Private sector & 79.9 & 8229.5 & \multirow{2}{*}{$\begin{array}{l}\text { Use of } \\
\text { Mentoring }\end{array}$} & Private sector & 79.38 & 8255 \\
\hline & Public sector & 77.29 & 4173.5 & & \begin{tabular}{|l|} 
Public sector \\
\end{tabular} & 79.74 & 4306 \\
\hline \multirow{3}{*}{$\begin{array}{l}\text { Use of } \\
\text { Development } \\
\text { centers }\end{array}$} & \begin{tabular}{|l} 
Private sector \\
\end{tabular} & 82.28 & 8474.5 & \multirow{3}{*}{$\begin{array}{l}\text { Use of e- } \\
\text { learning }\end{array}$} & $\begin{array}{l}\text { Private sector } \\
\end{array}$ & 85.14 & 8769.5 \\
\hline & Public sector & 72.75 & 3928.5 & & Public sector & 67.29 & 3633.5 \\
\hline & & & & & & & \\
\hline
\end{tabular}

Source: Authors' analysis based on Cranet database 
Table 5: Mann - Whitney U test and Means for differences between private and public organizations in career development practice

\begin{tabular}{|c|c|c|c|c|c|c|c|}
\hline \multicolumn{8}{|c|}{ Test Statistics $^{\text {a }}$} \\
\hline & $\begin{array}{l}\text { Use of } \\
\text { Special } \\
\text { tasks }\end{array}$ & $\begin{array}{l}\text { Use of } \\
\text { Projects to } \\
\text { stimulate } \\
\text { learning }\end{array}$ & $\begin{array}{c}\text { Use of } \\
\text { Training } \\
\text { on-the-job }\end{array}$ & $\begin{array}{c}\text { Use of } \\
\text { Participation } \\
\text { in project } \\
\text { team work }\end{array}$ & $\begin{array}{c}\text { Use of } \\
\text { Formal } \\
\text { networking } \\
\text { schemes }\end{array}$ & $\begin{array}{c}\text { Use of } \\
\text { Formal } \\
\text { career } \\
\text { plans }\end{array}$ & $\begin{array}{c}\text { Use of } \\
\text { Development } \\
\text { centres }\end{array}$ \\
\hline Private sector mean & 1.63 & 1.26 & 3.07 & 1.94 & 1.23 & 1.24 & .77 \\
\hline Public sector mean & 1.24 & 1.09 & 2.56 & 1.83 & 1.07 & 1.20 & .44 \\
\hline Total mean & 1.50 & 1.21 & 2.89 & 1.90 & 1.18 & 1.23 & .66 \\
\hline Mann-Whitney U & 2304.000 & 2488.000 & 2118.000 & 2668.500 & 2556.500 & 2688.500 & 2443.500 \\
\hline Asymp. Sig. (2-tailed) & .069 & .293 & .010 & .671 & .378 & .716 & .133 \\
\hline & $\begin{array}{c}\text { Use of } \\
\text { Succession } \\
\text { plans }\end{array}$ & $\begin{array}{c}\text { Use of } \\
\text { Planned } \\
\text { job rotation }\end{array}$ & $\begin{array}{c}\text { Use of } \\
\text { "High flier" } \\
\text { schemes }\end{array}$ & $\begin{array}{c}\text { Use of } \\
\text { International } \\
\text { work } \\
\text { assignments }\end{array}$ & $\begin{array}{c}\text { Use of } \\
\text { Coaching }\end{array}$ & $\begin{array}{c}\text { Use of } \\
\text { Mentoring }\end{array}$ & $\begin{array}{l}\text { Use of e- } \\
\text { learning }\end{array}$ \\
\hline Private sector mean & 1.43 & 1.26 & .99 & 1.17 & 1.57 & 2.79 & 1.38 \\
\hline Public sector mean & .81 & .62 & .32 & .46 & .78 & 2.81 & .74 \\
\hline Total mean & 1.22 & 1.04 & .76 & .93 & 1.30 & 2.80 & 1.16 \\
\hline Mann-Whitney U & 2041.500 & 1895.500 & 1937.500 & 1999.000 & 1881.000 & 2795.000 & 2148.500 \\
\hline Asymp. Sig. (2-tailed) & .004 & .001 & .001 & .001 & .000 & .960 & .012 \\
\hline
\end{tabular}

Source: Authors' analysis based on Cranet database

Table 6: Differences between private and public organizations in compensation practice

\begin{tabular}{|c|c|c|c|c|c|}
\hline & \multicolumn{2}{|c|}{ Flexible benefits } & \multirow{2}{*}{$\begin{array}{l}\text { Pearson Chi-Square } \\
\text { Asymp. Sig. (2-sided) }\end{array}$} & \multirow{2}{*}{\begin{tabular}{|l|}
4.177 \\
.041
\end{tabular}} \\
\hline & & Generally not used & Yes & & \\
\hline \multirow{2}{*}{ Sector } & Private sector & $82.9 \%$ & $17.1 \%$ & Phi & .162 \\
\hline & Public sector & $94.4 \%$ & $5.6 \%$ & & \\
\hline \multicolumn{2}{|l|}{ Total } & $86.8 \%$ & $13.2 \%$ & & \\
\hline & & \multicolumn{2}{|c|}{ Individual performance related pay } & Pearson Chi-Square & .851 \\
\hline & & Generally not used & Yes & Asymp. Sig. (2-sided) & .356 \\
\hline \multirow{2}{*}{ Sector } & Private sector & $33.3 \%$ & $66.7 \%$ & Phi & .073 \\
\hline & Public sector & $40.7 \%$ & $59.3 \%$ & & \\
\hline \multicolumn{2}{|l|}{ Total } & $35.8 \%$ & $64.2 \%$ & & \\
\hline & & \multicolumn{2}{|c|}{ Bonus based on individual goals } & Pearson Chi-Square & 22.759 \\
\hline & & Generally not used & Yes & Asymp. Sig. (2-sided) & .000 \\
\hline \multirow{2}{*}{ Sector } & Private sector & $43.8 \%$ & $56.2 \%$ & Phi & .378 \\
\hline & Public sector & $83.3 \%$ & $16.7 \%$ & & \\
\hline \multicolumn{2}{|l|}{ Total } & $57.2 \%$ & $42.8 \%$ & & \\
\hline & & \multicolumn{2}{|c|}{ Bonus based on organizational goals } & Pearson Chi-Square & 7.309 \\
\hline & & Generally not used & Yes & Asymp. Sig. (2-sided) & .007 \\
\hline \multirow{2}{*}{ Sector } & Private sector & $58.1 \%$ & $41.9 \%$ & Phi & .214 \\
\hline & Public sector & $79.6 \%$ & $20.4 \%$ & & \\
\hline \multicolumn{2}{|l|}{ Total } & $65.4 \%$ & $34.6 \%$ & & \\
\hline & & \multicolumn{2}{|c|}{ Non-monetary incentives } & Pearson Chi-Square & 5.351 \\
\hline & & Generally not used & Yes & Asymp. Sig. (2-sided) & .021 \\
\hline \multirow{2}{*}{ Sector } & Private sector & $55.2 \%$ & $44.8 \%$ & Phi & .183 \\
\hline & Public sector & $74.1 \%$ & $25.9 \%$ & & \\
\hline \multicolumn{2}{|l|}{ Total } & $61.6 \%$ & $38.4 \%$ & & \\
\hline
\end{tabular}

Source: Authors' analysis based on Cranet database

For the analysis of the differences between organizations from private and public sector in the usage of contemporary compensation techniques the authors used Spearman Chi Square test. According to the data presented in table 6, there are statistically significant differences between private and public organizations in the usage of pay for performance methods. Generally, organizations from public sector use modern techniques of rewarding professional workers less than those from the private sector $(p<0.05)$. This is obvious for all techniques of compensation, except for individual performance based pay, where there were no statistically significant $(\mathrm{p}=0.356)$ differences found. The strengths of the associations between these two variables were weak (Phi from 0.162 to 0.378 ).

We used Mann-Whitney $U$ test to explore the differences between the organizations from private and public sector regarding the trade union practice. According to the data cited in table 7 and 8 , there are statistically significant differences between private and public sector regarding the 
proportion of employees that are members of a trade union and the extent to which trade unions influence organization $(\mathrm{p}<0.05)$. Public sector organizations use trade union participation in a greater extent than organizations from the private sector (Means). Also, trade unions have greater power (the extent of the influence) in organizations from public $(M=3.3)$ than from private sector $(\mathrm{M}=1.69)$.

Table 7: Differences between private and public organizations in trade union practice

\begin{tabular}{|l|l|c|c|}
\hline & Sector & Mean Rank & Sum of Ranks \\
\hline \multirow{2}{*}{ Proportion of employees that are members of a trade union } & Private sector & 66.08 & 6938.50 \\
\cline { 2 - 4 } & Public sector & 107.06 & 5781.50 \\
\hline \multirow{2}{*}{ Extent to which trade unions influence organization } & Private sector & 66.72 & 7005.50 \\
\cline { 2 - 4 } & Public sector & 105.82 & 5714.50 \\
\hline
\end{tabular}

Source: Authors' analysis based on Cranet database

Table 8: Mann - Whitney U test and Means for differences between private and public organizations in trade union practice

\begin{tabular}{|l|c|c|}
\hline \multicolumn{2}{|c|}{ Test Statistics $^{\mathbf{a}}$} \\
\hline & $\begin{array}{c}\text { Proportion of employees that are members of a } \\
\text { trade union (from 1=0\%-10\% to 7=75\%-100\%) }\end{array}$ & $\begin{array}{c}\text { Extent to which trade unions } \\
\text { influence organization (from 1 to 5) }\end{array}$ \\
\hline Private sector mean & 3.00 & 1.69 \\
\hline Public sector mean & 4.98 & 3.30 \\
\hline Total mean & 3.67 & 2.23 \\
\hline Mann-Whitney U & 1373.500 & 1440.500 \\
\hline Wilcoxon W & 6938.500 & 7005.500 \\
\hline Z & -5.488 & -5.412 \\
\hline Asymp. Sig. (2-tailed) & .000 & .000 \\
\hline \multicolumn{2}{|l|}{ a. Grouping Variable: Sector } & \\
\hline
\end{tabular}

Source: Authors' analysis based on Cranet database

Table 9: Differences between private and public organizations in trade union practice

\begin{tabular}{|l|l|c|c|}
\hline \multirow{2}{*}{ Direct from senior managers } & Sector & Mean Rank & Sum of Ranks \\
\hline \multirow{2}{*}{ Through immediate superior } & Private sector & 80,12 & 8413,00 \\
\cline { 2 - 4 } & Public sector & 79,76 & 4307,00 \\
\hline \multirow{2}{*}{ Through trade union representatives } & Private sector & 78,84 & 8199,50 \\
\cline { 2 - 4 } & Public sector & 80,77 & 4361,50 \\
\hline \multirow{2}{*}{ Through works council to communicate with employees } & Private sector & 69,31 & 7208,00 \\
\cline { 2 - 4 } & Public sector & 99,13 & 5353,00 \\
\cline { 2 - 4 } & Private sector & 77,18 & 8026,50 \\
\hline \multirow{2}{*}{ Through regular workforce meetings } & Public sector & 82,58 & 4376,50 \\
\cline { 2 - 4 } & Private sector & 87,50 & 9012,00 \\
\hline \multirow{2}{*}{ Team briefings } & Public sector & 62,80 & 3391,00 \\
\cline { 2 - 4 } & Private sector & 85,48 & 8975,00 \\
\hline \multirow{2}{*}{ Electronic communication to communicate with employees } & Private sector & 69,35 & 3745,00 \\
\cline { 2 - 4 } & Public sector & 87,57 & 9107,50 \\
\hline \multirow{2}{*}{ Sour } & 63,95 & 3453,50 \\
\hline
\end{tabular}

Source: Authors' analysis based on Cranet database

Table 10: Mann - Whitney U test and Means for differences between private and public organizations in communication practice

\begin{tabular}{|l|c|c|c|c|c|c|c|}
\hline \multicolumn{9}{|c|}{ Test Statistics } & $\begin{array}{c}\text { a } \\
\text { Direct from } \\
\text { senior } \\
\text { managers }\end{array}$ & $\begin{array}{c}\text { Through } \\
\text { immediate } \\
\text { superior }\end{array}$ & $\begin{array}{c}\text { Through trade } \\
\text { union } \\
\text { representatives }\end{array}$ & $\begin{array}{c}\text { Through } \\
\text { works } \\
\text { council }\end{array}$ & $\begin{array}{c}\text { Through } \\
\text { regular } \\
\text { workforce } \\
\text { meetings }\end{array}$ & $\begin{array}{c}\text { Team } \\
\text { briefings }\end{array}$ & $\begin{array}{c}\text { Electronic } \\
\text { communication }\end{array}$ \\
\hline Private sector mean & 2,74 & 3,36 & 1,29 &, 38 & 2,67 & 2,22 & 2,93 \\
\hline Public sector mean & 2,81 & 3,41 & 2,26 &, 51 & 1,89 & 1,65 & 2,22 \\
\hline Total mean & 2,77 & 3,37 & 1,62 &, 42 & 2,40 & 2,03 & 2,69 \\
\hline Mann-Whitney U & 2822,000 & 2739,500 & 1748,000 & 2566,500 & 1906,000 & 2260,000 & 1968,500 \\
\hline $\begin{array}{l}\text { Asymp. Sig. } \\
\text { (2-tailed) }\end{array}$ &, 961 &, 769 & $\mathbf{, 0 0 0}$ &, 311 & $\mathbf{, 0 0 1}$ &, $\mathbf{0 3 1}$ &, $\mathbf{0 0 1}$ \\
\hline a. Grouping Variable: Sector & & & & \\
\hline
\end{tabular}

Source: Authors' analysis based on Cranet database 
We used Mann-Whitney $U$ test to explore the differences between organizations from private and public sector regarding the communication practice, i.e. how organizations communicate with their employees. According to the data shown in table 9 and 10, there are statistically significant differences between private and public sector regarding communication practice $(\mathrm{p}<0.05)$. Private sector organizations use modern communication channels (team briefings, ecommunication) to a greater extent than organizations from the public sector (Means). In contrast, public sector organizations use trade unions as a communication channel with their employees more than organizations from the private sector.

\section{CONCLUSION}

Based on the results of the research we can conclude that in Serbia human resource management approach in public sector is less developed than in private sector organization. A smaller share of public sector organizations use modern techniques for recruitment, selection, and compensation. Also, public sector organizations spend less money and days on training programs for their employees. In the case of communication, those organizations usually use traditional channels, direct from supervisors or via trade union representatives. Regarding the level of unionization and the influence of trade unions, as expected, public sector organizations have stronger trade union influence. In comparison with private sector organizations, we found that in Serbia HRM in public sector organization is less developed than in private sector organizations. The results of Spearman's Chi Square test and Mann-Whitney U test showed that smaller share of public sector organizations uses modern techniques of HRM than private sector organizations. These differences are statistically significant, so we can conclude that our hypothesis $\mathrm{H} 1$ is confirmed.

In order to improve its organizational performances, in the line with the idea of New Public Management, public sector organizations need to change its view on HRM. According to the results, HRM in public sector is still implemented as transactional practice, not like strategic function. To improve management practice a very important step will be the employment of high qualified HR managers and managers on the other levels in public sector organizations, their continuous training, and development, and strengthening of their cooperation in order to be successful in the implementation of HR strategies and practices. Generally, this can be seen as managerial professionalization in public management.

\section{REFERENCES}

Berber, N., \& Slavić, A. (2014). The practice of career development in the international human resource management of the European countries. Journal of Engineering Management and Competitiveness, 4(1), 21-26.

Boubakri, N., \& Cosset, J. C. (1998). The financial and operating performance of newly privatized firms: Evidence from developing countries. The Journal of Finance, 53(3), 1081-1110.

Boyne, G., Poole, M., \& Jenkins, G. (1999). Human resource management in the public and private sectors: An empirical comparison. Public Administration, 77(2), 407-420.

Brown, K. A. (2008). Human resource management in the public sector. In: R.S. Beattie \& S.P.Osborne (Ed.), Human resource management in the public sector, (pp. 1-7). London: Routledge.

Caemmerer, B., \& Dewar, A. (2013). A Comparison Of Private And Public Sector Performance. Journal of Applied Business Research, 29(5), 1451-1458.

Campbell, B. A., Coff, R., \& Kryscynski, D. (2012). Rethinking sustained competitive advantage from human capital. Academy of Management Review, 37(3), 376-395.

Chaston, I. (2011). Public Sector Management: Mission Imposible?. London: Palgrave MacMillan.

Giauque, D., Anderfuhren-Biget, S., \& Varone, F. (2013). HRM practices, intrinsic motivators, and organizational performance in the public sector. Public Personnel Management, 42(2), 123-150.

Gould-Williams, J. (2004). The effects of 'high commitment' HRM practices on employee attitude: the views of public sector workers. Public Administration, 82(1), 63-81.

Hughes, O.E. (2003). Public Management and Administration: An Introduction, $3^{\text {rd }}$ Edition. New York: Palgrave Macmillan.

Karoliny, Z., Farkas, F., \& Poór, J. (2009). In focus: Hungarian and Central Eastern European characteristics of human resource management - An international comparative survey. Journal of East European Management Studies, 14(1), 9-47.

Lazarova, M., Morley, M., \& Tyson, S. (2008). International comparative studies in HRM and performance-the Cranet data: Introduction. The International Journal of Human Resource Management, 19(11), 1995-2003.

Leković, B., Slavić, A., \& Berber, N. (2015). Praksa upravljanja ljudskim resursima u Srbiji 2014/2015 Izveštaj Cranet istraživanja. Subotica: Ekonomski fakultet. 
Marčetić, G. (2006). Upravljanje ljudskim potencijalima u javnoj upravi i novi Zakon o državnim službenicima. Hrvatska i komparativna javna uprava, 6(2), 83-118.

Mijić, K., Jakšić, D., \& Berber, N. (2015). The analysis of productivity and profitability in the CEE region: Focus on the private and public sector. Serbian Journal of Management, 10(2), 215-224.

Megginson, W. L., Nash, R. C., \& Randenborgh, M. (1994). The financial and operating performance of newly privatized firms: An international empirical analysis. The Journal of Finance, 49(2), 403-452.

Štangl Šušnjar, G. (2013). Menadžment urbanom sredinom. Subotica: Ekonomski fakultet.

Štangl Šušnjar, G., Slavić, A., Berber, N., \& Leković, B. (2016). The role of human resource management in small and medium sized companies in CentralEastern Europe. In J. Ateljević \& J. Trivić (Eds.), Economic Development and Entrepreneurship in
Transition Economies - Issues (pp. 205-229).

Switzerland: Springer International Publishing

Steinmetz, H., Schwens, C., Wehner, M., \& Kabst, R. (2011). Conceptual and methodological issues in comparative HRM research: The Cranet project as an example. Human Resource Management Review, 21(1), 16-26.

Urbano, D., \& Yordanova, D. (2008). Determinants of the adoption of HRM practices in tourism SMEs in Spain: an exploratory study. Service Business, 2(3), 167-185.

Wright, P. M., \& McMahan, G. C. (2011). Exploring human capital: putting 'human'back into strategic human resource management. Human Resource Management Journal, 21(2), 93-104.

Zolak-Poljašević, B., \& Petković, S. (2013). Human resource management in small and medium-sized enterprises: Conceptual framework. Facta universitatis-series: Economics and Organization, 10(3), 301-315.

\title{
HRM U PRIVATNIM I JAVNIM ORGANIZACIJAMA U SRBIJI
}

\begin{abstract}
Menadžment ljudskih resursa (Human Resource Management, HRM) je koncept upravljanja koji obuhvata različite funkcije i aktivnosti. Iako postoje značajni izvori literature o menadžmentu ljudskih resursa u privatnom sektoru, HRM praksa u javnom sektoru je i dalje nedovoljno istražena. U privatnom sektoru HRM se prihvata kao faktor za sticanje konkurentske prednosti, naročito ako HRM sprovodi kroz radne aktivnosti koje vode ka visokim performansama (High Performance Working Practices). U javnom sektoru menadžment ljudskim resursima se shvata kao paternalistički menadžment, sa standardizacijom prakse zapošljavanja, kolektivnog pregovaranja i radnih praksi koje naglašavaju jednake mogućnosti za sve zaposlene. Cilj ovog istraživanja je istraživanje karakteristika i razlika između HRM prakse u organizacijama iz privatnog i javnog sektora. Predmet istraživanja jesu aktivnosti menadžmeta ljudskih resursa (stafing, obuka i razvoj, kompenzacije i beneficije, radni odnos i komunikacija) u privatnim i javnim organizacijama u Republici Srbiji. Metodologija rada obuhvata istraživanje dostupne literature o temi i statističku analizu razlika između HR prakse u organizacijama iz privatnog i javnog sektora. Istraživanje je zasnovano na podacima prikupljenim u drugom CRANET istraživačkom periodu u Srbiji, iz 2015. godine.
\end{abstract}

Ključne reči: Menadžment ljudskih resursa, Privatni sektor, Javni sektor, Srbija, Cranet. 